Antonie van Leeuwenhoek

\title{
Genome analysis suggests the bacterial family Acetobacteraceae is a source of undiscovered specialized metabolites
}

Juan Guzman ${ }^{1, *}$, Andreas Vilcinskas ${ }^{1,2}$

1 Department of Bioresources, Fraunhofer Institute for Molecular Biology and Applied Ecology, Ohlebergsweg 12, D-35392 Giessen, Germany

2 Institute for Insect Biotechnology, Justus-Liebig-University of Giessen, Heinrich-Buff-Ring 26-32, D-35392, Giessen, Germany

Corresponding author:

Juan Guzman

juan.guzman@ime.fraunhofer.de

Telefon +496419937761

Fax +496414808581

\section{ORCID:}

Juan Guzman (0000-0003-4120-9065)

Andreas Vilcinskas (0000-0001-8276-4968)

\begin{abstract}
Acetobacteraceae is an economically important family of bacteria that is used for industrial fermentation in the food/feed sector and for the preparation of sorbose and bacterial cellulose. It comprises two major groups: acetous species (acetic acid bacteria) associated with flowers, fruits and insects, and acidophilic species, a phylogenetically basal and physiologically heterogeneous group inhabiting acid or hot springs, sludge, sewage and freshwater environments. Despite the biotechnological importance of the family Acetobacteraceae, the literature does not provide any information about its ability to produce specialized metabolites. We therefore constructed a phylogenomic tree based on concatenated protein sequences from 127 type strains of the family and predicted the presence of small-molecule biosynthetic gene clusters (BGCs) using the antiSMASH tool. This dual approach allowed us to associate certain biosynthetic pathways with particular taxonomic groups. We found that acidophilic and acetous species contain on average $\sim 6$ and $\sim 3.4$ BGCs per genome, respectively. All the Acetobacteraceae strains encoded proteins associated involved in hopanoid biosynthesis, with many also featuring genes encoding type-1 and type-3 polyketide and non-ribosomal peptide synthases, and enzymes for aryl polyene, lactone and ribosomal peptide biosynthesis. Our in silico analysis indicated that the family Acetobacteraceae is a potential source of many undiscovered bacterial metabolites and deserves more detailed experimental exploration.
\end{abstract}




\section{Keywords:}

Acetobacteraceae; phylogeny; biosynthesis; specialized metabolites

\section{Declarations}

\section{Funding}

JG is the recipient of a Georg Forster research fellowship from the Alexander von Humboldt Foundation. AV acknowledges support from the Hessian State Ministry of Higher Education, Research, Science and the Arts

(HMWK) for the project "Animal Venomics" embedded in the LOEWE Center for "Translational Biodiversity Genomics".

\section{Availability of data and material}

Datasets generated during this study are available to interested readers on request.

\section{Authors' contributions}

JG performed the analysis, prepared the figures and wrote the first draft. AV revised and improved the manuscript.

\section{Acknowledgments}

JG acknowledges Dr. Ivan Coludar for initial support with the phylogenomic analysis. The authors are thankful to Dr. Pablo Cruz-Morales for valuable comments on the manuscript.

\section{Conflict of interest}

The authors declare no conflict of interest. 


\section{Introduction}

Acetobacteraceae is an economically important family of bacteria, with several strains used for industrial biotechnology applications including the commercial production of vinegar and fermented foods, bacterial cellulose, and sorbose, a key precursor of vitamin C (Lynch et al. 2019; Murooka 2016; Pappenberger and Hohmann 2014). The family is divided into two groups: acetous and acidophilic species (Hördt et al. 2020; Komagata et al. 2014). Acetous species are also known as acetic acid bacteria (AAB) and most can transform ethanol into acetic acid, although there are some exceptions such as Asaia spp. (Malimas et al. 2017). AAB are typically found in flowers, fruits and other sugary organs of plants, and in traditional vinegars and other fermentation products (Yamada 2016), although some have recently been shown to consistently associate with insects (Guzman et al. 2021; Li et al. 2015; Roh et al. 2008). Acidophilic species appear to be phylogenetically more distant from the AAB (Hördt et al. 2020), and show diverse phenotypes and adaptations, including acidophilic, neutrophilic, thermophilic and phototrophic characteristics (Komagata et al. 2014). This group has been isolated from paddy soils, acid or hot springs, soil crust, sludge, sewage, freshwater ponds, air-conditioning systems, and certain Roseomonas strains have even been isolated from human patients (Dé et al. 2004; Sievers and Swings 2015). The Acetobacteraceae currently includes 44 genera and 177 valid species, split into 19 genera and 97 species of $\mathrm{AAB}$, and 25 genera and 80 species of acidophilic bacteria (Parte et al. 2020). The family belongs to the order Rhodospirillales, class Alphaproteobacteria, and their closer siblings are the recently proposed families Stellaceae and Reyranellaceae, based on phylogenomic and phenotypic analysis (Hördt et al. 2020). Some species currently classified as acidophilic bacteria are likely to be assigned to new families in the future when more genomic data become available. No specialized metabolites $(<2 \mathrm{kDa})$ have been reported thus far from any member of the family Acetobacteraceae, but it remains an untapped potential source of natural products given that related taxa appear to carry tens of biosynthetic gene clusters (BGCs) based on wide genomic analysis (Mukherjee et al. 2017).

The production of specialized metabolites has been intensively studied in streptomycetes and myxobacteria because they are known producers of antibiotics. The production of metabolites depends on the ecological context, in which the synthesized compounds confer competitive advantages to the producer, overcoming the energy costs of maintaining the BGCs (Hoskisson and Fernández-Martínez 2018; Jensen 2016). BGCs often encode not only enzymes but also other essential complementary proteins such as assembly scaffolds, metabolite resistance factors and regulatory effectors. Computational methods have been developed to identify the presence of BGCs in the exponentially growing resource of microbial genomic data (Medema et al. 2021; Medema and Fischbach 2015). The standard tool for this purpose is antiSMASH, which interrogates the protein sequences encoded in the genomes for sequence similarity to a library of hidden Markov models extracted from core biosynthetic proteins (Medema et al. 2011). The cluster boundaries are expanded to include other nearby core proteins, and accessory proteins in the vicinity are detected (Blin et al. 2017b). The search is finalized by evaluating the similarity of the detected gene set to known BGCs. One of the main limitations of library-based genome mining is that it detects proteins similar to known biosynthetic proteins but excludes unknown proteins that might produce entirely new molecules (Blin et al. 2017a). Here we took 127 published genomes of Acetobacteraceae type strains and used them for phylogenetic analysis and genome mining in order to find correlations between cladistics and the conservation of certain specialized biosynthetic traits. Our results will 
help to focus discovery efforts on bacterial producers of novel metabolites with potential applications in the pharmaceutical and agrochemical industries.

\section{Materials and methods}

\section{Genomic dataset}

The genome sequences of 129 bacterial type strains (Supplementary Table 1) were downloaded from NCBI GenBank in November 2020. The dataset comprised all 127 genomes available for Acetobacteraceae type strains as well as two type species as outgroups. The dataset included 40 acidophilic strains and 87 acetous strains. The outgroup strains, namely Azospirillum lipoferum $59 \mathrm{~b}^{\mathrm{T}}$ and Skermanella aerolata $\mathrm{KACC} 11604^{\mathrm{T}}$, were included to root the phylogenetic tree.

\section{Phylogenetic analysis}

The protein sequences were annotated from the downloaded genomes using Prokka v1.14.5 (Seemann 2014). Homologous protein sequences between species were identified using hmmsearch v3.2 (Eddy 2011) with hidden Markov models defined in bcgTree v1.1.10 (Ankenbrand and Keller 2016). The hits were aligned using Muscle v3.8.31 (Edgar 2004) and the unaligned sequences were trimmed using Gblocks v0.91b (Talavera and Castresana 2007). A concatenated alignment of 50 homologous proteins present as single copies in all species was generated, comprising the sequences of DnaN, DnlJ, Era, Frr, GrpE, InfC, LepA, NusA, PrfA, PyrG, RbfA, RecA, SecY, L-Gly-tRNA and L-Thr-tRNA ligases, and ribosomal proteins L1, L2, L3, L4, L5, L6, L9, L10, L12, L13, L14, L16, L17, L19, L20, L23, L24, L27, L29, L28, S2, S3, S4, S7, S6, S8, S9, S11, S12, S15, S16, S17, S18, S19 and S20. The tree was inferred using ExaBayes v1.5.1 (Aberer et al. 2014) as a consensus model built from four independent runs estimated over $10^{6}$ generations. A tree with exactly the same topology and similar branch support values was generated from the alignment using IQ-Tree v2.1.3 (Minh et al. 2020). The best model search for each partitioned protein sequence was performed using ModelFinder (Kalyaanamoorthy et al. 2017) and bootstrap values were calculated over $10^{5}$ replicates using ultrafast bootstrap approximation (Hoang et al. 2017).

\section{Biosynthetic gene clusters}

BGCs were predicted on the same dataset of 127 Acetobacteraceae type strains. The downloaded genomes were analyzed using the antibiotics and secondary metabolite analysis shell (antiSMASH) v5.2.0 (Blin et al. 2019) website in relaxed mode with all the extra features selected. Each identified region was counted and classified according to the biosynthetic core and accessory genes. BLAST analysis of the protein sequences against the GenBank non-redundant protein sequences database (340.8 million entries) was used to infer functional annotations for certain core and accessory proteins. The files in gbk format were downloaded from antiSMASH and used to assess gene cluster synteny and protein-protein similarity using Clinker v0.0.12 (Gilchrist and Chooi 2020) based on the BGCs for known compounds in the MIBIG repository (Kautsar et al. 2019).

\section{Results and Discussion}

GC content and genome size

A graphic plot examination of the variation in GC-content and genome size values (Figure 1a) for the 129 type strains supported a rough separation of the family Acetobacteraceae into the acetous and acidophilic groups. The Acetobacteraceae genomes not classified as AAB (with the exception of Acidocella aminolytica $101^{\mathrm{T}}$ ) showed a 
narrow GC content range $(\Delta \sim 11 \% \mathrm{~mol})$ with values between $62.7 \% \mathrm{~mol}$ (Roseomonas cervicalis ATCC $49957^{\mathrm{T}}$ ) and $73.9 \% \mathrm{~mol}$ (Crenalkalicoccus roseus YIM 78023 ${ }^{\mathrm{T}}$ ). However, this group showed a large variation in genome size ( $\Delta \sim 4.8 \mathrm{Mbp}$ ), ranging from $3.03 \mathrm{Mbp}$ (Elioraea thermophila YIM $72297^{\mathrm{T}}$ ) to $7.78 \mathrm{Mbp}$ (Dankookia rubra JCM $\left.30602^{\mathrm{T}}\right)$. In contrast, the GC content of the AAB varied widely $(\Delta \sim 31 \%$ mol), with values between $36.8 \% \mathrm{~mol}$ (Commensalibacter intestini $\mathrm{A} 911^{\mathrm{T}}$ ) and $67.7 \% \mathrm{~mol}$ (Endobacter medicaginis LMG $\left.26838^{\mathrm{T}}\right)$. However, this group showed less variation in genome size $(\Delta \sim 2.8 \mathrm{Mbp})$, ranging from $2.01 \mathrm{Mbp}$ (Parasaccharibacter apium $\mathrm{A} 29^{\mathrm{T}}$ ) to $4.83 \mathrm{Mbp}$ (Gluconacetobacter sacchari LMG $19747^{\mathrm{T}}$ ). Interestingly, AAB genera isolated exclusively from the insect gut, consisting of the genera Bombella (= "Parasaccharibacter"), Commensalibacter and Entomobacter, clustered in a region of low genome size within the acetous group, suggesting an ongoing evolutionary reduction of genome size probably reflecting their symbiotic lifestyles.

\section{Phylogenomics}

Phylogenomic analysis based on protein sequences confirmed that the acetous group originated from a lineage, probably already inhabiting low-pH environments, derived from the more basal acidophilic group (Figure 1b). Acetobacteraceae type strains were organized into suprageneric or infrageneric clades (Figure $1 \mathrm{~b}$ and Supplementary Table 2) according to the position in the phylogenomic tree. Four distinct clades were recognized within the acidophilic group: the early separating branch containing the genus Elioraea, followed by an extensive group containing the genus Roseomonas and 10 related genera, the pool of strains representing Acidocella and Acidiphilium strains, and finally the lineages composed of Acidibrevibacterium, Rhodovastum, Acidisphaera and Rhodopila strains, which shared a late common ancestor with the acetous group. The proposed Roseomonas clade contained subgroups of species belonging to the genus Roseomonas with intercalated strains from the genera Belnapia, Dankookia, Humitaela, Paracraurococcus, Rhodovarius, Roseicella, Roseococcus, Rubritepida, Siccirubricoccus and Teichococcus. The polyphyletic origin of the genus Rosemonas sensu stricto suggests that it will be revised in the future. The topology of the acetous group confirmed the current accepted demarcation of most genera with a few exceptions. As previously suggested (Yamada et al. 2012), the type strain Gluconacetobacter entanii LTH $4560^{\mathrm{T}}$ belongs to the genus Komagataeibacter. The type strain Nguyenibacter vanlangensis $\mathrm{LMG} 31431^{\mathrm{T}}$ was positioned very close to the diazotrophicus clade of Gluconacetobacter (Figure 1b). N. vanlangensis LMG $31431^{\mathrm{T}}$ fixes nitrogen and thus benefits plants (Vu et al. 2019) in a similar manner to Gluconacetobacter diazotrophicus LMG $7603^{\mathrm{T}}$ (Reis and Teixeira 2015). Together with our results, these data suggest that the LMG $31431^{\mathrm{T}}$ strain should belong to the genus Gluconoacetobacter. Given the high-support nodes indicating late common ancestors within certain members of a same genus, we proposed subgroups (clades named according to the most ancient described type species) for the genera Acetobacter, Gluconacetobacter, Gluconobacter and Komagataeibacter (Figure 1b). The topology of the phylogenomic tree obtained in this study is in full agreement with an earlier report (Hördt et al. 2020).

\section{Biosynthesis of specialized metabolites}

The BGCs identified in the family Acetobacteraceae using antiSMASH were organized in 10 groups according to the metabolite class or pathway: arylpolyene, ectoine, lactone, type-1 polyketide synthase (PKS), type-3 PKS, ribosomally synthesized and post-translationally modified peptide (RiPP), siderophore, terpenoid, non-ribosomal peptide synthetase (NRPS), NRPS/PKS hybrids, and miscellaneous BGCs. All type strains of the family carried at least one BGC and the global average was 4.2 $\pm 2.3 \mathrm{BGCs} /$ genome. Members of the acidophilic group carried on average $6.0 \pm 2.6 \mathrm{BGCs} /$ genome, which was $\sim 1.8$ more than the members of the acetous group, which carried 
on average 3.4 $\pm 1.5 \mathrm{BGCs} /$ genome (Figure 2a). This difference was statistically significant $(\mathrm{p}<0.001)$ based on both the Kruskal-Wallis test and the Benjamini-Hochberg test. All the Acetobacteraceae type strains carried gene clusters involved in terpenoid biosynthesis. These BCGs were found on average at a frequency of $\sim 2.5$ BGCs/genome in the acidophilic group, and $~ 1.5 \mathrm{BGCs} /$ genome in the acetous group (Figure 2b). In general, the acidophilic group carried a larger number of class-specific BGCs than the acetous group, and this was particularly evident for type-1 and type-3 PKS genes (Figure 2b). The genomes of some strains featured four different PKS genes, for example Roseomonas stagni DSM $19981^{\mathrm{T}}$. NRPS genes were also prominently found in the genomes of the acidophilic group, particularly in the genera Roseomonas and Rhodovastum. The one BGC that was present in higher numbers in the acetous group was involved in the production of RiPPs (Figure 2b).

A direct correlation between taxonomy or phylogeny and the presence of certain types of BGCs was not evident, but certain trends were observed (Figure 3). For example, the acidophilic group generally carried some miscellaneous gene clusters for phosphonates and indoles, whereas no acetous species carried these BGCs (Figure 3ab). Most of the species ( 76\%) in the genus Roseomonas carried at least one polyketide cluster with the exception of $R$. mucosa NCTC $13292^{\mathrm{T}}, R$. rosea DSM $14916^{\mathrm{T}}, R$. aerophila NBRC $108923^{\mathrm{T}}$ and R. cervicalis ATCC $49957^{\mathrm{T}}$. All Gluconacetobacter species featured a type-1 PKS, whereas not a single strain of the related genus Komagataeibacter carried a polyketide cluster. Among the acetous group, the genus Acetobacter tended to feature more BGCs than others (Figure 3ac), with the highest numbers identified in the strains A. persici JCM 25330 (eight BGCs) and A. senegalensis LMG 23690 (seven BGCs). Acetobacter species were proficient in the biosynthesis of lactones and non-ribosomal peptides, whereas members of the Asaia-Bombella clade and certain Gluconobacter carried BGCs for the synthesis of polyketides.

\section{Aryl polyenes}

Aryl polyenes (APEs) are bacterial pigments produced abundantly by the phylum Proteobacteria, and like carotenoids these unsaturated molecules play a role in the capture of free radicals to prevent oxidative stress (Schöner et al. 2016). The biosynthesis of APEs involves the loading of an aromatic precursor (usually 4-hydroxybenzoic acid) onto an acyl carrier protein (ACP), named ApeE, in a process catalyzed by ApeH (Grammbitter et al. 2020). The central enzyme $\beta$-ketoacyl-ACP synthase (ApeO/ApeC) elongates the chain in a decarboxylation Claisen condensation with malonate units, and the cetone product is reduced to alcohol by ApeQ and dehydrated to a double bond by ApI/ApeP in an iterative process. For some metabolites, the aryl polyene is linked to $\mathrm{N}$-acetylglucosamine by the glycosyltransferase ApeJ. The presence of homologs of the core $\beta$-ketoacyl-ACP synthase among Acetobacteraceae allowed the identification of APE producers. The acidophilic group featured a higher proportion $(16 / 40=40 \%)$ of APE gene clusters than the acetous group $(23 / 87=26 \%)$, suggesting that bacteria readily exposed to sunlight, such as those inhabiting ponds, probably produce APEs for protection against $\mathrm{UV}$ radiation.

The $\beta$-ketoacyl-ACP synthase encoded in the genomes of Gluconacetobacter sacchari LMG $19747^{\mathrm{T}}$, Nguyenibacter vanlangensis LMG $31431^{\mathrm{T}}$ and Swaminathania salitolerans NBRC $104436^{\mathrm{T}}$ differed from the other homologs in the family (Supplementary Figure 1a), and were probably transferred horizontally from other organisms (particularly from Gammaproteobacteria, given that homologous proteins were identified in Yersinia, Serratia and Pseudomonas). Some genes linked to the $\beta$-ketoacyl-ACP synthase gene were tentatively annotated as encoding an adenylate-forming protein, a dehydrogenase, a probable halogenase, and transport proteins, 
whereas others were hypothetical. The aryl polyene BCGs of the family Acetobacteraceae appear likely to produce as yet undescribed aryl polyene products.

\section{Ectoines}

Ectoines are bacterial natural products sharing a 4-carboxylic acid pyrimidine that promote survival in hyperosmotic environments (Czech et al. 2018). Ectoines are synthesized from L-aspartate- $\beta$-semialdehyde by the sequential action of three proteins: EctB, EctA and EctC (Czech et al. 2018). The final enzyme (EctC) is known as L-ectoine synthase, and catalyzes the transformation of $N_{4}$-acetyl-L-2,4-diaminobutanoate to L-ectoine, acting as a marker for the identification of ectoine BGCs. Homologs of this EctC protein in Paenibacillus lautus NBRC 15380 (Czech et al. 2019) were detected in only five Acetobacteraceae type strains: Acidocella aminolytica $101^{\mathrm{T}}$, Acidiphilium cryptum $\mathrm{JF}-5^{\mathrm{T}}$, Acidiphilium multivorum AUI301 ${ }^{\mathrm{T}}$, Acetobacter nitrogenificens DSM $23291^{\mathrm{T}}$ and Gluconobacter wancherniae NBRC $103581^{\mathrm{T}}$ (Supplementary Figure 1b). The three members of the acidophilic group have a full ectoine cluster, including genes encoding other enzymes in the pathway such as EctA, EctB, EctD and the L-aspartate kinase (Ask) together with a transporter and a transcriptional regulator. Interestingly, the clusters in the two acetous strains with an ectC gene carried no linked homologs of ectA, ectB, $e c t D$ or $a s k$, and thus it is uncertain whether the EctC protein is a functional L-ectoine synthase or has a different role. An EctA homolog was identified in A. nitrogenificens DSM $23291^{\mathrm{T}}$ but not in G. wancherniae NBRC $103581^{\mathrm{T}}$. Homologs of the $N$-acetyltransferase EctB were detected in both strains but also in many other Acetobacter and Gluconobacter species, suggesting involvement in a more general pathway. Finally, no EctD homologs were detected in any species of the acetous group. These results suggest that the acetous group probably does not produce ectoines, and the functional role of EctC homologs in A. nitrogenificens DSM $23291^{\mathrm{T}}$ and $G$. wancherniae NBRC $103581^{\mathrm{T}}$ should be investigated in more detail.

\section{Hopanoids}

The most common protein sequence encoded in the Acetobacteraceae terpenoid clusters was used as a blastp query, resulting in the identification of an enzyme involved in hopene biosynthesis. Hopanoids are pentacyclic bacterial triterpenoids that confer fluidity and integrity to the cell membrane in a similar manner to sterols (such as cholesterol and sitosterol) in eukaryotes (Sáenz et al. 2015). All Acetobacteraceae type strains carried genes for hopanoid synthesis. The most common hopanoid BGC consisted of genes for squalene-hopene cyclase (SHC) and two squalene synthases. This type of cluster was often found in the acetous group, and hopanoids may therefore protect the cell membrane against injury caused by acetic acid (Belin et al. 2018; Welander et al. 2009). In the acidophilic group, these biosynthetic genes were not clustered together and typically one or more was missing. SHC is the central enzyme of hopanoid biosynthesis and is responsible for the cascade polycyclization of squalene leading to the pentacyclic hopene triterpenoid (Siedenburg and Jendrossek 2011). Certain species of Acetobacter, Gluconacetobacter, Gluconobacter and Komagataeibacter encoded two versions of the SHC protein (Supplementary Figure 1c), and although most Acetobacteraceae carried the most common SHC, some carried only the second type. This second SHC shared a consensus sequence of $\sim 30$ amino acids near the C-terminus that is not present in the major SHC or in homologous proteins from Streptomyces but is found in some species of the genera Zymomonas, Bradyrhizobium and Rhodopseudomonas. Homologs of the two versions of SHC found in certain Acetobacteraceae also occur in Zymomonas mobilis and their activity has been verified experimentally (Seitz et al. 2012). The three-gene hopanoid core BGC of the acetous group also contained additional genes for accessory proteins, the most common of which were annotated as a glycosyltransferase, a 
FAD-dependent oxidoreductase, and a NAD-dependent epimerase/hydratase. In a number of species of Roseomonas (such as R. aerilata DSM19363 $R$. nepalensis $\mathrm{S} 9^{\mathrm{T}}$ and $R$. oryzae $\mathrm{KCTC} 42542^{\mathrm{T}}$ ), the core hopene cyclase gene was linked to a phosphoenolpyruvate mutase gene, which is the marker for organophosphonic acid synthesis (Horsman and Zechel 2017), suggesting it is part of a hybrid cluster that generates a yet unknown compound. In Acetobacter malorum LMG $1746^{\mathrm{T}}$, the alternative hopene cyclase gene was linked to an auto-inducer synthase gene, indicating that further natural hopanoids with yet unknown functions may exist.

\section{Lactones}

Two types of lactone BGCs were found in the Acetobacteraceae genomes, encoding the enzymes needed for the production of acyl-homoserine lactones (AHLs) and $\beta$-lactones, respectively. AHLs are involved in quorum sensing (QS), an intercellular communication process that triggers coordinated gene expression (Waters and Bassler 2005). AHLs are QS auto-inducing factors because they bind to a transcription factor (LuxR in Vibrio fischerii) which activates the expression of the gene encoding the AHL synthase (LuxI in V. fischerii), resulting in the massive production of AHLs throughout the population. LuxR and LuxI homologs are widespread in Proteobacteria (Case et al. 2008; Schuster et al. 2013). AHLs are produced from $S$-adenosylmethionine by cleavage, cyclization and $N$-acylation with an ACP or acyl-coenzyme A (Schaefer et al. 2018). Genes for AHL biosynthesis were identified in only three species of the acidophilic group: Acidocella aminolytica $101^{\mathrm{T}}$ (two clusters), Acidibrevibacterium fodinaquatile $\mathrm{G} 45-3^{\mathrm{T}}$ and Roseomonas nepalensis $\mathrm{S} 9-3 \mathrm{~B}^{\mathrm{T}}$. However, they were found more frequently in the acetous group (22/87), being present in Nguyenibacter vanlangensis LMG $31431^{\mathrm{T}}$, four species of Gluconacetobacter, four species of Komagataeibacter and 13 species of Acetobacter. Some Acetobacter species, especially those strains belonging to the related orleanensis and tropicalis clades (Supplementary Figure 1d), carried two copies of the luxI/R cluster. Detailed analysis of protein alignments of the auto-inducer synthases (LuxI homologs) revealed three major groups, two of them specific for Acetobacter, and the third shared between Komagataeibacter and Gluconacetobacter. Some strains of Acetobacter (such as A. cerevisiae $\mathrm{LMG} 1625^{\mathrm{T}}$, A. malorum LMG $1746^{\mathrm{T}}$, A. orientalis $21 \mathrm{~F}-2^{\mathrm{T}}$, and A. persici $\mathrm{JCM} 25330^{\mathrm{T}}$ ) carried both types of auto-inducer synthases, suggesting the importance of QS in certain AABs used for the fermentation of must, fruit and cereal (Guillamón and Mas 2009; Iida et al. 2008; Valera et al. 2016). Interestingly the autoinducer synthase from $N$. vanlangensis $\mathrm{LMG} 31431^{\mathrm{T}}$ was clustered with the synthases from Gammaproteobacteria (Vibrio and Pseudomonas) and may originate by horizontal transfer. The protein sequence of the auto-inducer synthases from the acidophilic group were distantly related to those identified in the acetous group. Particularly those found in A. fodinaquatile $\mathrm{G} 45-3^{\mathrm{T}}$ and $R$. nepalensis $\mathrm{S} 9-3 \mathrm{~B}^{\mathrm{T}}$ showed a large branch length in the phylogenomic tree (Supplementary Figure 1d), suggesting the existence of an alternative QS route that should be investigated in future experiments.

BGCs responsible for $\beta$-lactone biosynthesis were not identified in the acetous group but were found in eight of the acidophilic type strains, all belonging to the Roseomonas clade. Seven species of Roseomonas and Belnapia rosea $\mathrm{CGMCC} 1.10758^{\mathrm{T}}$ carried a single $\beta$-lactone $\mathrm{BGC}$. Three $\beta$-lactone core enzymes were encoded by these clusters: a $\beta$-lactone AMP-binding protein supposedly catalyzing the coupling of a carboxylic acid (such as acetate) to coenzyme A, an HGML-like protein catalyzing the Claisen condensation of the acyl-CoA with a carboxylic acid to produce a $\beta$-ketoacid, and a dehydrogenase that reduces the intermediate to a $\beta$-hydroxyacid (Robinson et al. 2019). The final cyclization to the $\beta$-lactone is carried out by an ATP-dependent cyclase homologous to OleC (Robinson et al. 2019), but such a protein was not encoded by any of the BGCs. It is 
therefore unclear whether the product of these clusters is a $\beta$-lactone or a $\beta$-hydroxyacid. The $\beta$-hydroxyacid product may be a precursor in another specialized pathway, given that the $\beta$-lactone cluster in some Roseomonas strains (such as $R$. pecuniae DSM $25622^{\mathrm{T}}$ and $R$. vastitatis CPCC $1121^{\mathrm{T}}$ ) is fused with an NRPS cluster.

\section{Polyketides}

We identified type-1 and type-3 PKS genes in the Acetobacteraceae type strains. Type-1 PKS genes encode large proteins organized into modules that use ACPs to activate acyl-CoA substrates, whereas type-3 PKS genes encode products that act directly on acyl-CoA substrates and often produce cyclized aromatic polyketides (Jenke-Kodama et al. 2005; Shen 2003). We detected a type-1 PKS in more than half of the acidophilic species (23/48) and in one third of the acetous species (29/87). The PKS genes were found in specific taxonomic groups such as the Roseomonas clade (Figure 3ab), both clades of Gluconacetobacter, as well as Asaia and BombellaSaccharibacter and in certain species of Acetobacter and Gluconobacter. For a yet unknown reason, type-1 PKS genes were not found in the genus Komagateibacter. The high degree of PKS conservation in the different AAB clades rules out horizontal transfer and suggests that the resulting metabolites conferred advantages on the common ancestor and remain beneficial to the extant species in their current ecological context.

A basic motif found in most Acetobacteraceae type-1 PKS proteins consisted of the ordered domains KS-ATDH-ER-KR-PP (ketosynthase-acyltransferase-dehydratase-enoylreductase-ketoreductase-phosphopantheteine acyl carrier). In each strain, this basic motif was accompanied by a variety of small domains including aminotransferases (AmT), AMP-binding domains (A), coenzyme A-binding domains (CAL), enoyl-CoA hydratase/isomerase domains (ACH), NAD-dependent epimerase/dehydratase domains (NAD), further KR or ER domains, and/or a combination of such domains. Intriguingly thioesterase domains could not be identified within the PKS protein or as stand-alone accessory proteins. In all cases, the KS domains clustered with the type1 PKS gene, such as those associated with the synthesis of aureothin or certain aromatic polyketides (Chen and Du 2016). The presence of a single module suggests that the Acetobacteraceae PKS system is iterative and not modular. The PKS amino acid sequence is considered a good proxy to infer the number of metabolic products. The unrooted tree of Acetobacteraceae type-1 PKS proteins based on sequence alignment revealed four different clades, which we named $\alpha, \beta, \gamma$ and $\delta$ (Figure 4a). The $\alpha$-group included all PKS proteins from the Roseomonas clade, except the second PKS of Roseomonas stagnii DSM $19981^{\mathrm{T}}$ and the PKS from Rubritepida flocculans DSM $14296^{\mathrm{T}}$, both of which clustered in the $\delta$-group together with Acidiphilium angustum ATCC $35903^{\mathrm{T}}$. The $\alpha$-group could be divided into two subgroups, one containing all the Roseomonas strains (with the exception of Humitalea rosea DSM $24525^{\mathrm{T}}$ ) and the second including the non-Roseomonas strains (Figure 4a). In all the $\alpha$ group, a glycosyltransferase gene probably belonging to family GT4 (Breton et al. 2005), was found upstream of the PKS gene (Figure 4b). The $\alpha$-group also included genes for a PLP-dependent aminotransferase, a formyltransferase and a capsular biosynthetic protein. The metabolite produced by these $\alpha$-group type-1 PKS clusters is anticipated to have the same skeleton decorated with small variations given the different accessory proteins encoded within each cluster. The $\beta$-group included PKS proteins from the genera Acetobacter and Gluconobacter, and a branching group leading to the Asaia and Bombella clades (Figure 4a). The $\beta$-group type-1 PKS clusters differed from $\alpha, \gamma$ and $\delta$ clusters given the absence of a PLP-dependent aminotransferase gene, and instead the PKS gene was flanked by acyl ligase genes (Figure 4c). An $O$-heptosyltransferase gene was also consistently found in the $\beta$-group clusters, and was duplicated in the Bombella clade and the Gluconobacter spp. clusters. In addition, the PKS cluster from the Bombella clade was closely related to the cluster found in 
Swaminathania salitolerans NBRC $104436^{\mathrm{T}}$ and both clusters shared the presence of an additional glycosyltransferase and a thioredoxin, suggesting they may produce sulfur-containing metabolites. The $\gamma$-group was restricted to members of the genus Gluconacetobacter, and intriguingly these PKS proteins were more closely related to those from the acidophilic group rather than the rest of the acetous group (Figure 4a) The $\gamma$ group type-1 PKS clusters (Figure 4d) were highly conserved in gene organization and protein sequence, and probably synthesize the same metabolite, perhaps with the exception of G. tumulisoli LMG $27802^{\mathrm{T}}$. These clusters encoded a PLP-dependent aminotransferase, two capsular biosynthetic proteins and two glycosyltransferases. A small number of type-1 PKS proteins from acidophilic species clustered in the $\delta$-group (Figure 4a), and the BGCs (Figure 4e) encoded a PLP-dependent aminotransferase, a phosphatidyltransferase and an oxidoreductase, located near the central type-1 PKS gene.

A type-3 PKS was identified in 18 of the 48 acidophilic type strains (37.5\%) but none was found in any of the acetous species. This correlates with a specific evolutionary niche within plants for this group of bacteria, where probably the metabolic product of the type-3 PKS cluster is unnecessary for adaptation to the phytosphere. A type-3 PKS was found in several strains of the general Roseomonas $(13 / 28=46 \%)$, Acidocella $(3 / 5=60 \%)$ and Rhodopila $(2 / 4=50 \%)$. The closest sequences beyond the Acetobacteraceae were identified in other Alphaproteobacteria, including Azospirillium, Methylopila, Microvirga and Paracoccus species. A similar type-3 PKS is ArsC (sequence identity 29\%) from Azotobacter vinelandii strain OP, which produces alkylresorcinols and alkylpyrones to protect its cysts against environmental injury (Funa et al. 2006). The type-3 PKS proteins from the acidophilic group are therefore likely to be involved in pyrone or resorcinol biosynthesis, and may also play a protective role because this group of bacteria thrives in sediments, soils, ponds and hot springs (Komagata et al. 2014) where solar radiation and desiccation can be detrimental. Type-3 PKS proteins from the family Acetobacteraceae could be assigned to three groups based on sequence alignment and phylogeny (Supplementary Figure 2a). Specifically, we observed the divergence of Roseomonas frigidaquae JCM 15073 ${ }^{\mathrm{T}}$ and Roseomonas stagni DSM $19981^{\mathrm{T}}$ (closer to Belnapia and Siccirubricoccus than to the main Roseomonas group). In addition to the central type-3 PKS, two other proteins were encoded in all the clusters: a methyltransferase and a FAD-dependent monooxygenase (Supplementary Figure 2b). Interestingly, more closely related homologs of the methyltransferase were identified in other Rhodospirillales, such as Azospirillium, Indioceanicola and Skermanella species, but also in the myxobacterium Sorangium cellulosum, a recognized producer of specialized metabolites (Schneiker et al. 2007). The presence of methyltransferases and flavindependent monoxygenases is a common feature of certain type-3 PKS clusters particularly those found in fungi (Navarro-Muñoz and Collemare 2020) and in some myxobacteria (Hug et al. 2019), but the metabolite produced by Rhodospirillales is currently unknown.

\section{NRPS and hybrid NRPS/PKS clusters}

NRPS genes were present in 45\% (18/40) of the acidophilic species, and some strains featured multiple NRPS or NRPS-like clusters such as Roseomonas aerophila NBRC $198923^{\mathrm{T}}$ with four. The NRPS clusters were much less common among the acetous species, being present in only $\sim 14 \%(12 / 87)$. Like PKS genes, NPRS genes encode megasynthases organized into modules, including condensation (C), adenylation (A), thiolation (also known as peptidyl carrier protein, PCP), and thioesterase (TE) domains. Like the ACP in PKS, the PCP is activated by the transfer of a 4'-phosphopantetheine factor. Among the acidophilic species, eight of the 27 NRPS genes were trimodular, six were tetramodular, five were monomodular, four were bimodular, and two each were 
pentamodular and hexamodular. In contrast, nine of the NRPS genes in the acetous group were monomodular, three were bimodular, one trimodular and one hexamodular. The lower number and complexity of NRPS clusters among the acetous species may probably reflect genome reduction related to plant speciation events. The lack of these specific NRPS clusters in both Acetobacteraceae clades exclusive to insects (Bonilla-Rosso et al. 2019) is consistent with this hypothesis, and suggests that such peptides are probably more important for bacteria living in soil, sediment or water environments.

Only a few species carried complete C-A-PCP-TE domains in a single protein (Supplementary Figure 2c). In the acetous group, only two closely related Acetobacter species (A. malorum LMG $1746^{\mathrm{T}}$ and $A$. cibinongensis NBRC $16605^{\mathrm{T}}$ ) featured these domains in a single monomodular NRPS, whereas A. persici JCM $25330^{\mathrm{T}}$ and A. aceti NBRC $14818^{\mathrm{T}}$ featured NPRS genes with the C-A-PCP-TE domains split into adjacent modules and also contained further modules with AmT and CAL domains, which are more common in PKS genes. Two strains of Komagataeibacter carried an NRPS-like cluster (K. diospyri MSKU9 ${ }^{\mathrm{T}}$ and $K$. swingsii LMG 22125 ${ }^{\mathrm{T}}$ ), but in both cases the NRPS gene contained A, PCP and TE domains, but no apparent C domain, suggesting either that $\mathrm{C}$ domains are provided by non-canonical hypothetical genes or that the cluster does not express a functional NRPS product and may be involved in other biosynthesis reactions (or may be the product of translocation or recombination events). Similar NRPS-like genes encoding A, PCP and TE but not C domains were found in Roseomonas deserti $\mathrm{M}^{\mathrm{T}}$ and Roseomonas vastitatis CPCC $1011021^{\mathrm{T}}$ but their organization and the composition of accessory genes was different. Complete C-A-PCP-TE domains in a single module were also observed in trimodular clusters from Roseomonas frigidaquae JCM $15073^{\mathrm{T}}$ and Rhodovastum atsumiense DSM $21279^{\mathrm{T}}$, in tetramodular clusters from Roseomonas rhizosphaerae $\mathrm{YW} 11^{\mathrm{T}}$ and Roseomonas rosea DSM $14916^{\mathrm{T}}$, in a pentamodular cluster from Roseomonas aestuarii JR169-1-13 ${ }^{\mathrm{T}}$, and in a hexamodular cluster from Roseomonas mucosa $\mathrm{NCTC} 13291^{\mathrm{T}}$. The accompanying modules may provide adenylation or AMT, KR and ECH domains, which are most often found in PKS clusters, or a combination of these. Moreover, most of the NRPS clusters from the acidophilic group did not possess the complete minimal set of C-A-PCP-TE domains, and it is unclear if functional peptides are produced by these clusters. It is possible that non-canonical NRPS domains not detected using our algorithms are encoded in hypothetical accessory proteins. Multimodular NRPS clusters with repetitive C-A and KR domains, respectively, were identified in Acetobacter senegalensis LMG $23690^{\mathrm{T}}$ and Komagataeibacter rhaeticus LMG $22126^{\mathrm{T}}$.

Chimeric or hybrid NRPS-PKS clusters with contiguous PKS and NRPS modules were identified in Belnapia rosea CGMCC $1.10758^{\mathrm{T}}$, Roseomonas stagni DSM $19981^{\mathrm{T}}$ and Siccirubricoccus deserti SYSU D8009 ${ }^{\mathrm{T}}$. These three hybrid clusters were unique among the Acetobacteraceae type strains and they are likely to produce undiscovered metabolites, which deserve further detailed study. An almost identical hybrid cluster present in Asaia bogorensis NBRC $16594^{\mathrm{T}}$ and Asaia astilbis JCM $15831^{\mathrm{T}}$ encoded two megasynthases (one PKS and one NRPS) in opposing reading directions. Gene expression in these clusters is probably regulated by a histidine kinase receptor. The NRPS amino acid sequence has some similarity to vicibactin VbsS from Rhizobium spp. (Heemstra et al. 2009), and this megasynthase may similarly catalyze the trimerization of certain amino acid residues.

Ribosomally synthetized and post-translationally modified peptides 
RiPP gene clusters were identified in a handful of acidophilic strains including Acidocella facilis ATCC $35904^{\mathrm{T}}$, Rhodopila globiformis DSM 161 ${ }^{\mathrm{T}}$, Rhodovastum atsumiense DSM 21279, Roseomonas aestuarii JR169-1-13 ${ }^{\mathrm{T}}$ and Roseomonas mисоsa NCTC $13291^{\mathrm{T}}$. In contrast, such clusters were much more prevalent in the acetous group (Figure 2b), being present in all Komagataeibacter strains, all Gluconacetobacter strains except Gluconacetobacter johannae LMG 21312 ${ }^{\mathrm{T}}$, in 70\% (19/27) of the Acetobacter strains, and in four Gluconobacter strains (Figure 3ab). Interestingly, no RiPP clusters were found in Asaia species or in the Saccharibacter-Bombella clades. The only insect-associated AAB type strain carrying a RiPP cluster was Entomobacter blattae $\mathrm{G}_{5} \mathrm{GP}^{\mathrm{T}}$, which is predicted to produce a yet unknown linear azol(in)e peptide.

Roseomonas mucosa NCTC $13291^{\mathrm{T}}$ was the only species to also carry a BGC encoding a YcaO cyclohydratase, which catalyzes ring formation in azol(in)e peptides. Finally, a cyanobactin peptidase gene involved in the final step of RiPP maturation was found in Roseomonas oryzae $\mathrm{KCTC} 42542^{\mathrm{T}}$, and it is likely that this strain produces a new cyanobactin-like peptide.

The RiPP clusters found in the acidophilic group (except Roseomonas mucosa NCTC 13291 ${ }^{\mathrm{T}}$ ) encoded a DUF692-like protein homologous to MbnB from Methylosinus trichosporium OB3b, which binds iron and forms a complex with $\mathrm{MnbC}$ to catalyze the formation of an oxazolone-thioamide group on the core peptide sequence of methanobactin, a copper-chelating molecule (Kenney et al. 2018). In those species with a DUF692-like cluster, we did not identify a leader sequence or homologs of $\mathrm{MnbC}$ or the TonB receptor. However, this leadercore peptide sequence along with $\mathrm{MnbC}$ and TonB homologs were identified in the acetous group. Accordingly, Acetobacter oryzoeni $\mathrm{B}^{\mathrm{T}}$, Gluconacetobacter asukensis LMG $27724^{\mathrm{T}}$, Komagataeibacter nataicola $\mathrm{LMG}$ $1536^{\mathrm{T}}$, K. rhaeticus $\mathrm{LMG} 22126^{\mathrm{T}}$ and K. xylinus $\mathrm{LMG} 1515^{\mathrm{T}}$ are likely to produce as yet uncharacterized molecules related to methanobactins. The core DUF692 protein encoded by A. oryzoeni B6 $^{\mathrm{T}}$, K. rhaeticus LMG $22126^{\mathrm{T}}$ and K. xylinus LMG $1515^{\mathrm{T}}$ had exactly the same sequence. An unrooted tree based on the DUF692 protein agreed well with the existing phylogeny, clearly distinguishing the acetous and acidophilic groups (Supplementary Figure 2d), and intriguingly the protein from G. asukensis LMG $27724^{\mathrm{T}}$ was located in the acidophilic cluster. Rhodopila globiformis DSM $161^{\mathrm{T}}$ featured two RiPP clusters, the standard DUF692-like cluster and a second cluster encoding a TIGR03798-like protein probably involved in the synthesis of a yet unknown peptide.

The RiPP clusters found in acetous species can be assigned to two major groups: the DUF692 cluster also found in the acidophilic species and the linocin M18 cluster. The latter was exclusive to acetous species and was the most common cluster after the hopanoids, being present in $51(\sim 59 \%)$ of the acetous type strains. This cluster was present in all type strains of the genus Komagataeibacter and in $\sim 70 \%$ of the Acetobacter species. Gluconacetobacter tumulisoli LMG $27802^{\mathrm{T}}$ featured two linocin M18 clusters, and Gluconacetobacter aggeris LMG $27801^{\mathrm{T}}$ and Gluconacetobacter tumulicola LMG $27725^{\mathrm{T}}$ shared exactly the same core linocin M18 protein sequence. Intriguingly, the linocin M18 cluster was not found in any Asaia or Neokomagataea species, or in the Bombella-Saccharibacter and Ameyamaea-Tanticharoenia clades, suggesting this pathway is required for certain yet unknown ecological relationships with plants. The unrooted tree based on the linocin M18 protein (Supplementary Figure 2e) was interesting because there was no clear genus demarcation between Acetobacter, Komagataeibacter and Gluconacetobacter. This suggests evolution from a common ancestor with a certain degree of independence from taxonomic restrictions at the genus level, or recent horizontal gene transfer.

Notably, this cluster was not present in any of the insect-associated clades. Because none of the basal acidophilic 
strains can produce the linocin M18 biosynthetic protein, the ancestor protein in AAB was probably transferred from plant-dwelling members of the family Nitrobacteraceae such as Bradyrhizobium, given the presence of homologs in this genus. A very similar linocin M18 cluster has been studied in Rhodococcus jostii RHA1 and was found to encode a DypB peroxidase and an encapsulin protein that together generate a biochemically active lignin degradation nano-compartment (Rahmanpour and Bugg 2013). The linocin cluster found in AAB also encoded an encapsulin and a Dyp-type peroxidase, suggesting this cluster is involved in lignin degradation.

\section{Siderophores}

Siderophores are iron-scavenging metabolites that allow producers to thrive in iron-depleted environments. They are particularly useful for microbial competition and are considered virulence factors in pathogenic organisms (Miethke and Marahiel 2007). Only NRPS-independent pathways for siderophore biosynthesis (Oves-Costales et al. 2009) were identified in the Acetobacteraceae, particularly in the basal phylogenetic clades of the acetous group (6/87) and only in one strain of the acidophilic group (Figure 3ab). Two types of siderophore BGCs were identified. One cluster, shared by Dankookia rubra JCM $362^{\mathrm{T}}$ and Granulibacter bethesdensis CGDNIH1 ${ }^{\mathrm{T}}$, encoded two NRPS-independent siderophore synthases (IucA/IucC-like) (Supplementary Figure 2f), homologous to proteins from strains of the order Hyphomycrobiales (class Alphaproteobacteria) such as Methylobacterium, Pseudovibrio and Brucella spp. In addition, the cluster encoded an $\mathrm{N}$-acetyltransferase and a flavin-dependent lysine $N$-monooxygenase, and the resulting metabolite is probably a yet undescribed siderophore. The second cluster was shared by two Gluconacetobacter species (G. azotocaptans LMG $21311^{\mathrm{T}}$ and G. tumulicola LMG $27725^{\mathrm{T}}$ ) as well as Nguyenibacter vanlangensis LMG $31431^{\mathrm{T}}$, and encoded a single IucA/IucC-like synthase and for a number of proteins of unknown function (Supplementary Figure 2g). The siderophore cluster of E. blattae G55GP ${ }^{\mathrm{T}}$ is unique in the family Acetobacteraceae and the encoded proteins show homology to proteins from strains of the genera Azotobacter and Pseudomonas, and are distantly related to the clusters for vibrioferrin and xanthoferrin biosynthesis (Pandey et al. 2017; Tanabe et al. 2003). An IucA/IucC-like synthase was identified in the genome of Endobacter medicaginis LMG $26383^{\mathrm{T}}$ but the gene context remains unknown due to the large number of scaffolds.

\section{Miscellaneous biosynthetic clusters}

The acidophilic type strains also encoded biosynthetic proteins for less common specialized metabolites such as phosphonates and indoles, but such clusters were not present in the acetous species. Eight strains (20\%) in the acidophilic group encoded a homolog of phosphoenolpyruvate mutase and are likely to produce uncharacterized phosphonates. The presence of pyruvate decarboxylase and aminotransferase genes adjacent to the mutase indicated the formation of phosphonoacetaldehyde and finally 2-aminoethylphosphonate, which may be integrated into variety of end-products (Horsman and Zechel 2017). Two classes of phosphoryl mutase were identified in the clade, a shorter version present in Rhodovastum atsumiense DSM $21279^{\mathrm{T}}$ and Roseomonas oryzicola $\mathrm{KCTC} 22478^{\mathrm{T}}$, and the larger and most frequent version in Belnapia rosea CGMCC $1.10758^{\mathrm{T}}$ and five Roseomonas strains (Supplementary Figure 2h). Terpenoid biosynthesis genes were often closely linked to the mutase gene, suggesting that the product is an undiscovered terpene-phosphonate. $N$-acyl amino acids are synthesized from corresponding amino acid precursors by homologs of the $N$-acyl amino acid synthase NasY (Craig et al. 2011). Interestingly, NasY homologs were found exclusively in three type strains of the genus Acidiphilium, and this biosynthetic property is likely to be a marker of this genus. A putative homolog of PhzB, which catalyzes the synthesis of phenazine, was identified in the genome of Roseomonas vastitatis CPCC 
$1011021^{\mathrm{T}}$, but no other genes related to phenazine biosynthesis were found in the vicinity. PhzB is a member of the nuclear transport factor 2 (NTF2) family, which may have other functions in bacterial cells (Eberhardt et al. 2013), so it is not yet clear whether this strain can produce phenazines.

\section{Conclusion}

The family Acetobacteraceae belongs to the class Alphaproteobacteria, and members of this class are not generally considered prolific producers of specialized metabolites, despite some strains carrying more than forty BGCs (Mukherjee et al. 2017). A relatively small number of molecules have been characterized from this taxonomic class, however so far, no specialized metabolites $(<2 \mathrm{kDa})$ have been purified from strains of the family Acetobacteraceae. In this study we were able to predict that all members of the Acetobacteraceae are producers of hopanoids. These triterpenoids play a fundamental role in the integrity of the bacterial cell membrane, particularly under stressful conditions such as low $\mathrm{pH}$, but given the presence of two distinct hopanoid BGCs in a number of acetous species it is possible that these metabolites have additional functions. The acidophilic group featured almost twice as many BGCs as the acetous group. Most of the strains in both groups featured at least one type-1 PKS, and most members of the acidophilic group featured at least one NRPS and one type-3 PKS. The acetous group was found also to produce some ribosomal peptides. A smaller number of strains in both groups appear able to produce aryl polyenes, lactones and siderophores. Thus far, none of these metabolites has been purified, and the translation of metabolic potential in silico to actual metabolic capability remains to be confirmed. Given the diverse ecological niches occupied by the Acetobacteraceae, including ponds, sludge, soil, sediments, fruits, flowers and insect guts, the specialized metabolites produced by these species are likely to be bioactive and may be suitable for biotechnological exploitation. 

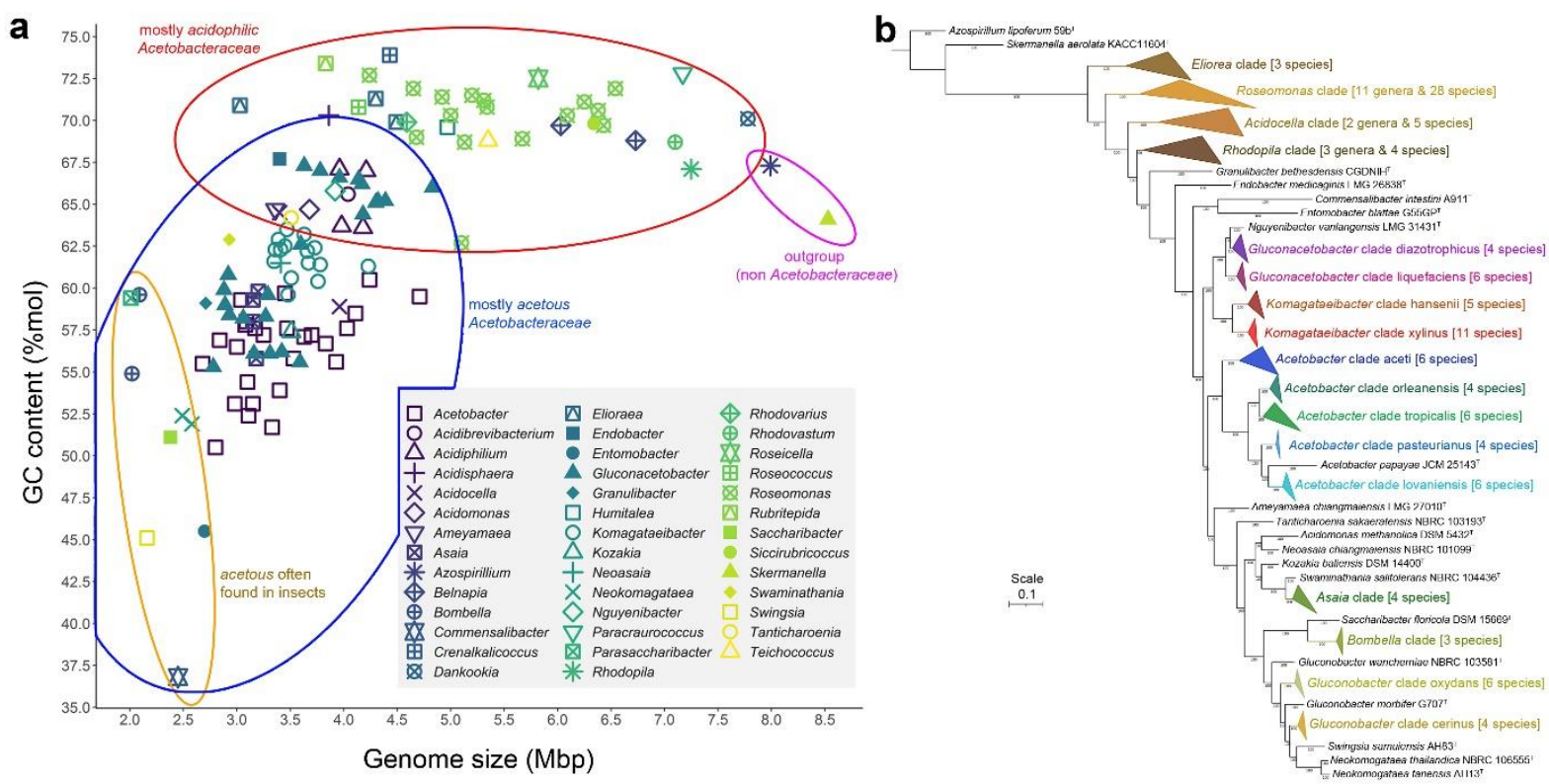

Figure 1. GC content $v$ s genome size plot and phylogenomic tree for Acetobacteraceae type strains a) GC content and genome size plot grouping the type strains from each genus under the same symbol. The strains Azospirillum lipoferum $59 \mathrm{~b}^{\mathrm{T}}$ and Skermanella aerolata $\mathrm{KACC} 11604^{\mathrm{T}}$ are used as outgroups for the family Acetobacteraceae. The plot reveals three groups of bacteria with some degree of overlap, globally differentiated as mostly acidophilic, mostly acetous, and acetous species often associated with insects. b) Phylogenomic tree inferred from 50 housekeeping protein sequences. The topology of the tree is supported by both Bayesian and maximum likelihood inference methods. Distinct clades (based on monophyly and a shorter branch length distance) were proposed particularly for the acetous group. The species organization into clades is detailed in Supplementary Table 2. 


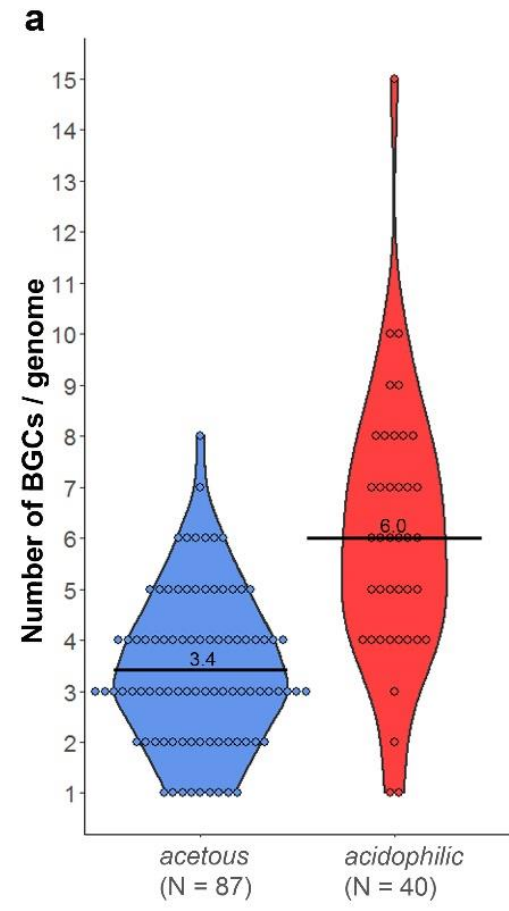

Acetobacteraceae groups

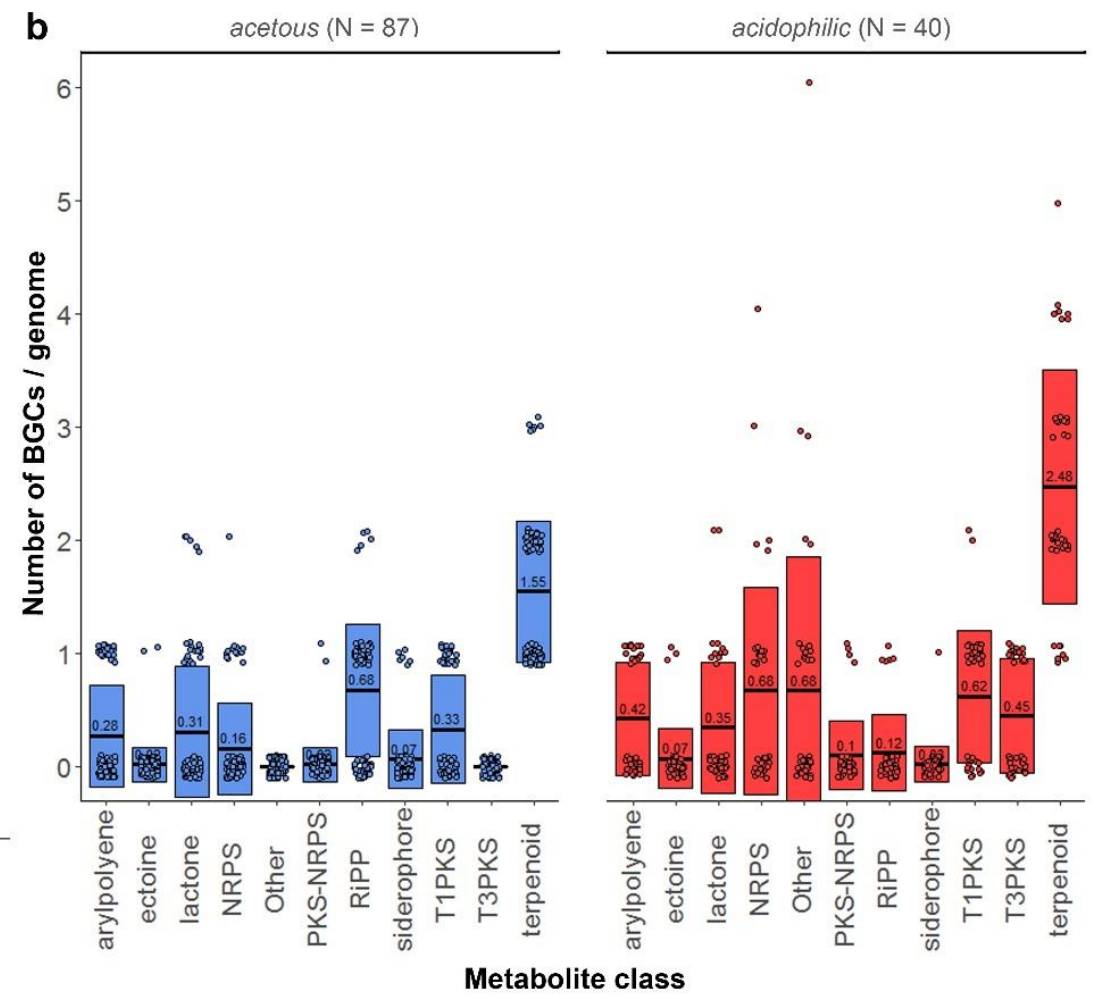

Figure 2. Presence of biosynthetic gene clusters (BGCs) in the two groups of the family Acetobacteraceae. a) The number of BGCs per genome was plotted for each type strain, organized according to the taxonomic classification into acetous and acidophilic species. b) BGCs for the biosynthesis of different metabolite classes were plotted for each type strain and were organized according to the taxonomic classification into acetous and acidophilic species. The numbers inside the boxplots are the calculated mean values. 
a

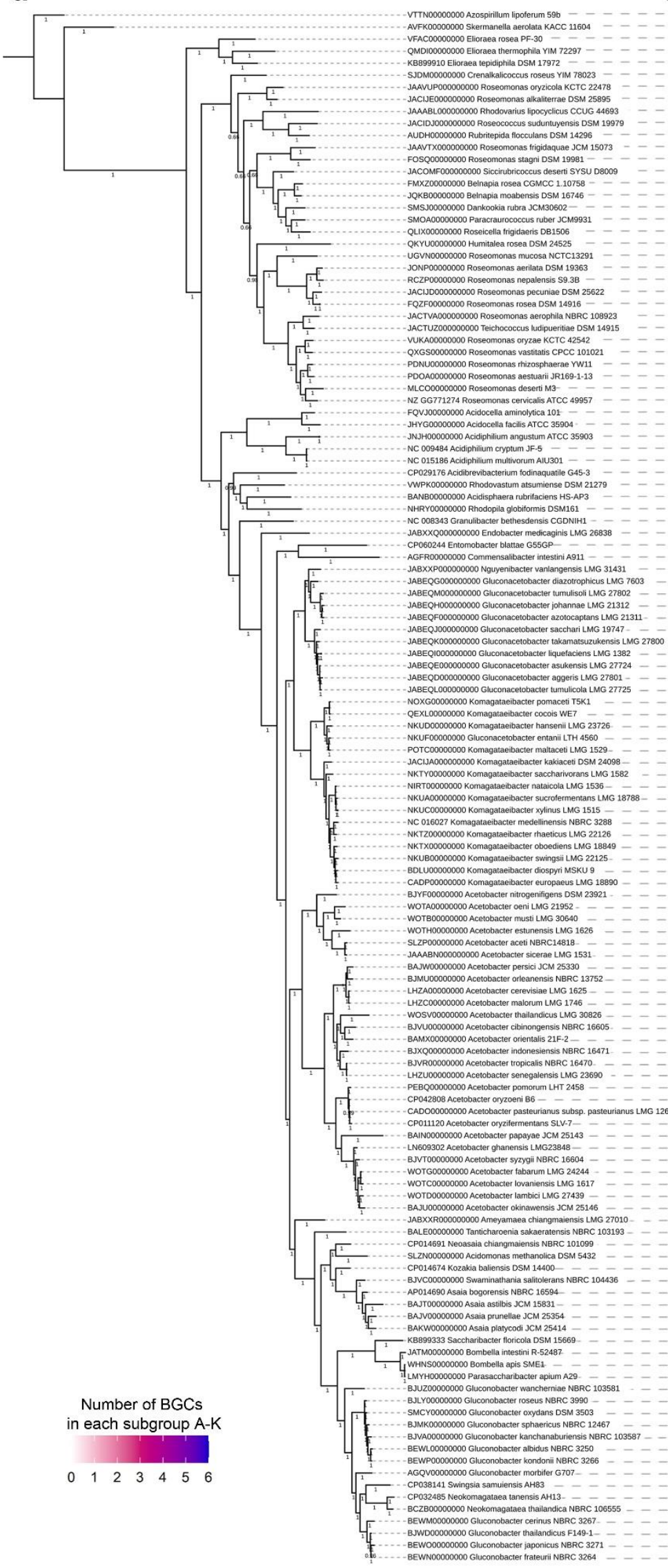

b

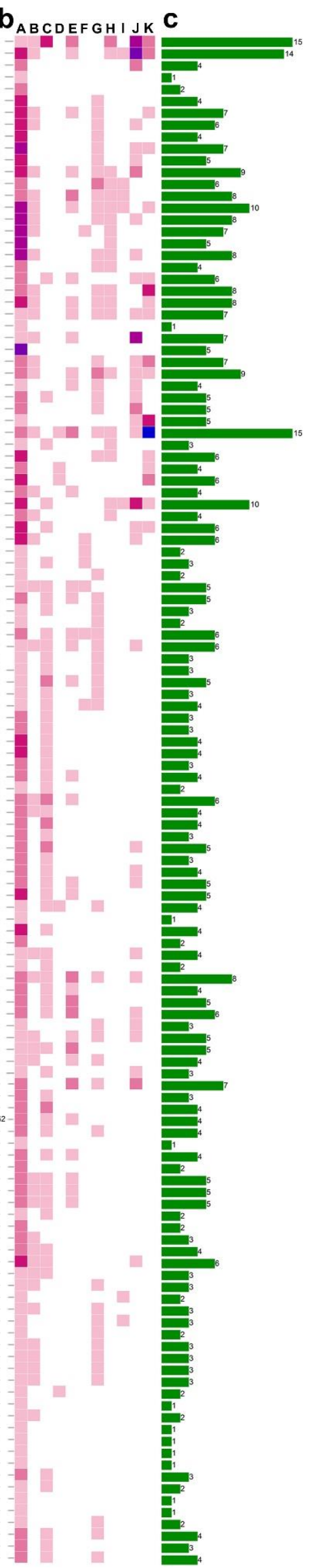

Figure 3. Phylogenomic analysis of the family Acetobacteraceae and their biosynthetic gene clusters (BGCs) as detected using antiSMASH. a) Phylogenomic tree based on 50 housekeeping protein sequences. b) Type and number of BCGs in the genomes of each type species. c) Total number of BCGs with at least one core 
gene detected using antiSMASH. The subgroups were classified according to the class or pathway of the metabolite as follows: $\mathrm{A}=$ terpenoid, $\mathrm{B}=$ aryl polyene, $\mathrm{C}=$ ribosomally synthesized and post-translationally modified peptide, $\mathrm{D}=$ ectoine, $\mathrm{E}=$ lactone, $\mathrm{F}=$ siderophore, $\mathrm{G}=$ type- 1 polyketide, $\mathrm{H}=$ type- 3 polyketide, $\mathrm{I}=$ hybrid polyketide/non-ribosomal peptide, $\mathrm{J}=$ non-ribosomal peptide, $\mathrm{K}$ = other specialized metabolites.

a

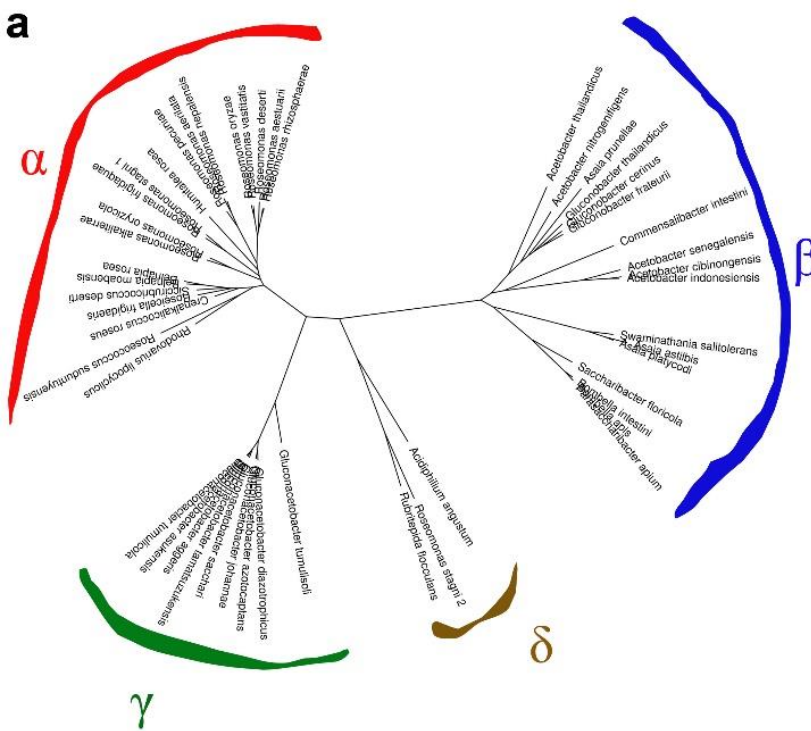

b

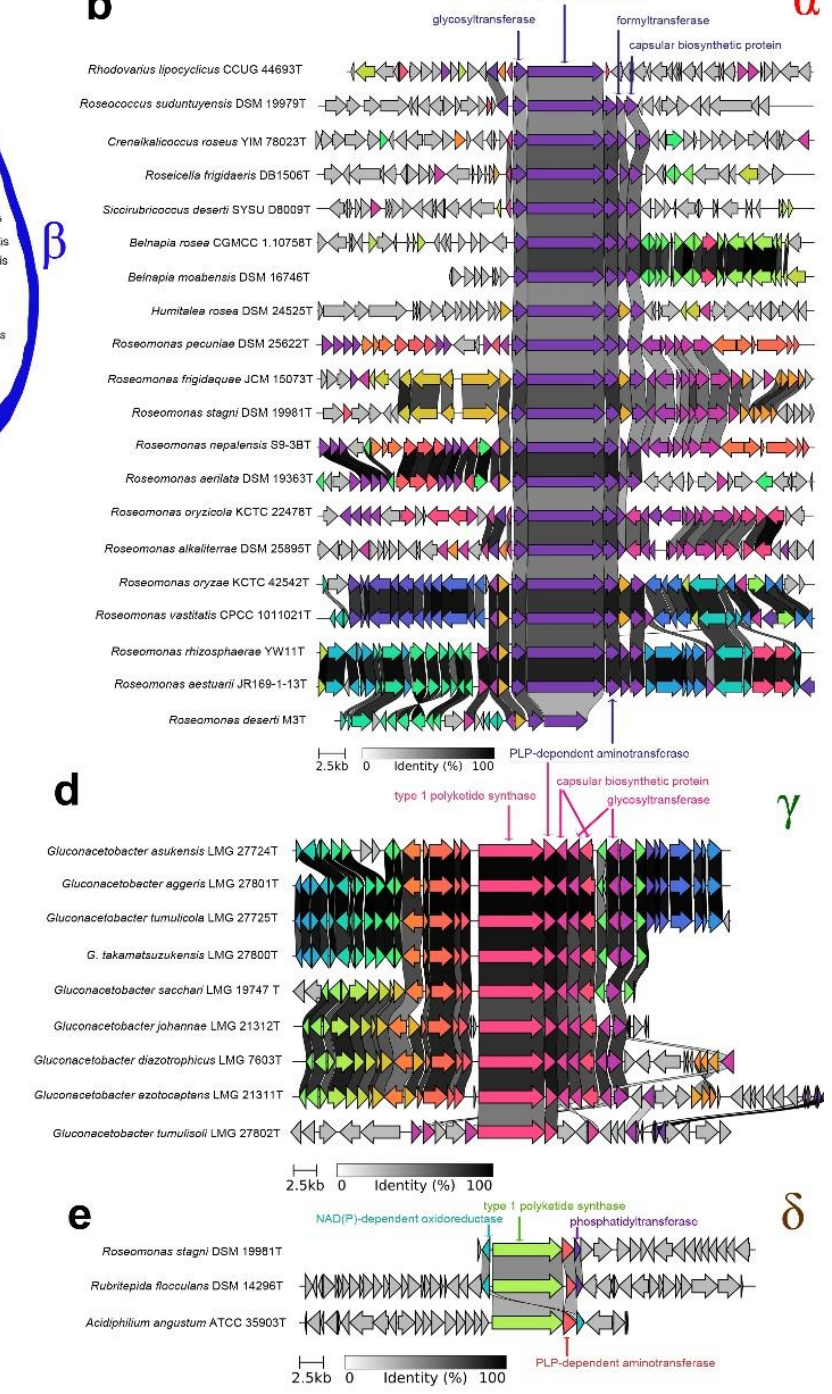

C

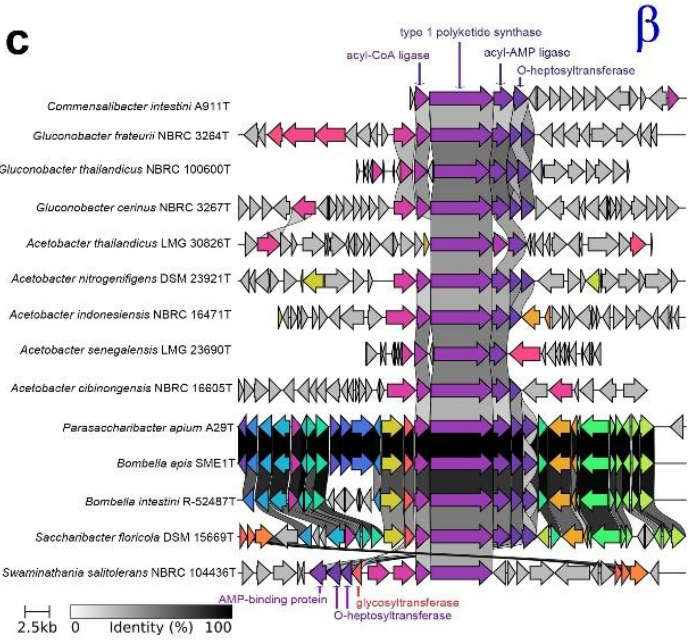

Figure 4. Type-1 polyketide synthase biosynthetic gene cluster in Acetobacteraceae. a) Unrooted tree based on type-1 PKS showing the differentiation into four groups labelled $\alpha, \beta, \gamma$ and $\delta$ which correlate with certain taxonomic clades. Organization of the biosynthetic gene clusters for the type-1 PKS from the groups $\alpha$ (b) $\beta$ (c) $\gamma$ (d) and $\delta(\mathbf{e})$ showing the probable annotation of certain genes according to antiSMASH and blast analysis. 


\section{References}

Aberer AJ, Kobert K, Stamatakis A (2014) ExaBayes: massively parallel bayesian tree inference for the wholegenome era. Mol Biol Evol 31:2553-2556 https://doi.org/10.1093/molbev/msu236

Ankenbrand MJ, Keller A (2016) bcgTree: automatized phylogenetic tree building from bacterial core genomes. Genome 59:783-791 https://doi.org/10.1139/gen-2015-0175

Belin BJ, Busset N, Giraud E, Molinaro A, Silipo A, Newman DK (2018) Hopanoid lipids: from membranes to plant-bacteria interactions. Nat Rev Microbiol 16:304-315 https://doi.org/10.1038/nrmicro.2017.173

Blin K, Kim HU, Medema MH, Weber T (2017a) Recent development of antiSMASH and other computational approaches to mine secondary metabolite biosynthetic gene clusters. Brief Bioinform 20:1103-1113 https://doi.org/10.1093/bib/bbx146

Blin K et al. (2019) antiSMASH 5.0: updates to the secondary metabolite genome mining pipeline. Nucleic Acids Res 47:W81-W87 https://doi.org/10.1093/nar/gkz310

Blin K et al. (2017b) antiSMASH 4.0-improvements in chemistry prediction and gene cluster boundary identification. Nucleic Acids Res 45:W36-W41 https://doi.org/10.1093/nar/gkx319

Bonilla-Rosso G et al. (2019) Acetobacteraceae in the honey bee gut comprise two distant clades with diverging metabolism and ecological niches. bioRxiv:861260 https://doi.org/10.1101/861260

Breton C, Šnajdrová L, Jeanneau C, Koča J, Imberty A (2005) Structures and mechanisms of glycosyltransferases. Glycobiol 16:29R-37R https://doi.org/10.1093/glycob/cwj016

Case RJ, Labbate M, Kjelleberg S (2008) AHL-driven quorum-sensing circuits: their frequency and function among the Proteobacteria. ISME J 2:345-349 https://doi.org/10.1038/ismej.2008.13

Chen H, Du L (2016) Iterative polyketide biosynthesis by modular polyketide synthases in bacteria. Appl Microbiol Biotechnol 100:541-557 https://doi.org/10.1007/s00253-015-7093-0

Craig JW, Cherry MA, Brady SF (2011) Long-chain N-acyl amino acid synthases are linked to the putative PEPCTERM/exosortase protein-sorting system in Gram-negative bacteria. J Bacteriol 193:5707-5715 https://doi.org/10.1128/JB.05426-11

Czech L et al. (2018) Role of the extremolytes ectoine and hydroxyectoine as stress protectants and nutrients: genetics, phylogenomics, biochemistry, and structural analysis. Genes 9:177

Czech L et al. (2019) Illuminating the catalytic core of ectoine synthase through structural and biochemical analysis. Sci Rep 9:364 https://doi.org/10.1038/s41598-018-36247-w

Dé I, Rolston KVI, Han XY (2004) Clinical significance of Roseomonas species isolated from catheter and blood samples: Analysis of 36 cases in patients with cancer. Clin Infect Dis 38:1579-1584 https://doi.org/10.1086/420824

Eberhardt RY, Chang Y, Bateman A, Murzin AG, Axelrod HL, Hwang WC, Aravind L (2013) Filling out the structural map of the NTF2-like superfamily. BMC Bioinformatics 14:327 https://doi.org/10.1186/1471-2105-14-327

Eddy SR (2011) Accelerated profile HMM searches. PLoS Comput Biol 7:e1002195 https://doi.org/10.1371/journal.pcbi.1002195

Edgar RC (2004) MUSCLE: multiple sequence alignment with high accuracy and high throughput. Nucleic Acids Res 32:1792-1797 https://doi.org/10.1093/nar/gkh340

Funa N, Ozawa H, Hirata A, Horinouchi S (2006) Phenolic lipid synthesis by type III polyketide synthases is essential for cyst formation in Azotobacter vinelandii. PNAS 103:6356-6361 https://doi.org/10.1073/pnas.0511227103

Gilchrist CLM, Chooi Y-H (2020) clinker \& clustermap.js: Automatic generation of gene cluster comparison figures. bioRxiv:2020.2011.2008.370650 https://doi.org/10.1101/2020.11.08.370650

Grammbitter GLC et al. (2020) The chemical structure of widespread microbial aryl polyene lipids. bioRxiv:2020.2012.2019.423268 https://doi.org/10.1101/2020.12.19.423268

Guillamón JM, Mas A (2009) Acetic acid bacteria. In: König H, Unden G, Fröhlich J (eds) Biology of Microorganisms on Grapes, in Must and in Wine. Springer Berlin Heidelberg, Berlin, Heidelberg, pp 31-46. https://doi.org/10.1007/978-3-540-85463-0_2

Guzman J, Sombolestani AS, Poehlein A, Daniel R, Cleenwerck I, Vandamme P, Vilcinskas A (2021) Entomobacter blattae gen. nov., sp. nov., a new member of the Acetobacteraceae isolated from the gut of the cockroach Gromphadorhina portentosa. Int J Syst Evol Microbiol 71:3 https://doi.org/10.1099/ijsem.0.004666

Heemstra JR, Walsh CT, Sattely ES (2009) Enzymatic tailoring of ornithine in the biosynthesis of the rhizobium cyclic trihydroxamate siderophore vicibactin. J Am Chem Soc 131:15317-15329 https://doi.org/10.1021/ja9056008

Hoang DT, Chernomor O, von Haeseler A, Minh BQ, Vinh LS (2017) UFBoot2: Improving the ultrafast bootstrap approximation. Mol Biol Evol 35:518-522 https://doi.org/10.1093/molbev/msx281

Hördt A et al. (2020) Analysis of 1,000+ Type-Strain Genomes Substantially Improves Taxonomic Classification of Alphaproteobacteria. Front Microbiol https://doi.org/10.3389/fmicb.2020.00468 
Horsman GP, Zechel DL (2017) Phosphonate biochemistry. Chem Rev 117:5704-5783 https://doi.org/10.1021/acs.chemrev.6b00536

Hoskisson PA, Fernández-Martínez LT (2018) Regulation of specialised metabolites in Actinobacteria expanding the paradigms. Environ Microbiol Rep 10:231-238 https://doi.org/10.1111/1758-2229.12629

Hug JJ, Panter F, Krug D, Müller R (2019) Genome mining reveals uncommon alkylpyrones as type III PKS products from myxobacteria. J Ind Microbiol Biotechnol 46:319-334 https://doi.org/10.1007/s10295018-2105-6

Iida A, Ohnishi Y, Horinouchi S (2008) Control of acetic acid fermentation by quorum sensing via Nacylhomoserine lactones in Gluconacetobacter intermedius. J Bacteriol 190:2546-2555 https://doi.org/10.1128/jb.01698-07

Jenke-Kodama H, Sandmann A, Müller R, Dittmann E (2005) Evolutionary implications of bacterial polyketide synthases. Mol Biol Evol 22:2027-2039 https://doi.org/10.1093/molbev/msi193

Jensen PR (2016) Natural products and the gene cluster revolution. Trends Microbiol 24:968-977 https://doi.org/10.1016/j.tim.2016.07.006

Kalyaanamoorthy S, Minh BQ, Wong TKF, von Haeseler A, Jermiin LS (2017) ModelFinder: fast model selection for accurate phylogenetic estimates. Nat Methods 14:587-589 https://doi.org/10.1038/nmeth.4285

Kautsar SA et al. (2019) MIBiG 2.0: a repository for biosynthetic gene clusters of known function. Nucleic Acids Res 48:D454-D458 https://doi.org/10.1093/nar/gkz882

Kenney GE et al. (2018) The biosynthesis of methanobactin. Science 359:1411-1416 https://doi.org/10.1126/science.aap9437

Komagata K, Iino T, Yamada Y (2014) The Family Acetobacteraceae. In: Rosenberg E, DeLong EF, Lory S, Stackebrandt E, Thompson F (eds) The Prokaryotes: Alphaproteobacteria and Betaproteobacteria. Springer Berlin Heidelberg, Berlin, Heidelberg, pp 3-78. https://doi.org/10.1007/978-3-642-30197$1 \_396$

Li L et al. (2015) Bombella intestini gen. nov., sp. nov., an acetic acid bacterium isolated from bumble bee crop. Int J Syst Evol Microbiol 65:267-273 https://doi.org/10.1099/ijs.0.068049-0

Lynch KM, Zannini E, Wilkinson S, Daenen L, Arendt EK (2019) Physiology of acetic acid bacteria and their role in vinegar and fermented beverages. Compr Rev Food Sci Food Saf 18:587-625 https://doi.org/10.1111/1541-4337.12440

Malimas T, Vu HTL, Muramatsu Y, Yukphan P, Tanasupawat S, Yamada Y (2017) Systematics of acetic acid bacteria. In: Ilkin Yucel Sengun (eds) Acetic Acid Bacteria: Fundamentals and Food Applications, CRC, Boca Raton, pp 3-43. https://doi.org/10.1201/9781315153490

Medema MH et al. (2011) antiSMASH: rapid identification, annotation and analysis of secondary metabolite biosynthesis gene clusters in bacterial and fungal genome sequences. Nucleic Acids Res 39:W339W346 https://doi.org/10.1093/nar/gkr466

Medema MH, de Rond T, Moore BS (2021) Mining genomes to illuminate the specialized chemistry of life. Nat Rev Gen https://doi.org/10.1038/s41576-021-00363-7

Medema MH, Fischbach MA (2015) Computational approaches to natural product discovery. Nat Chem Biol 11:639 https://doi.org/10.1038/nchembio.1884

Miethke M, Marahiel MA (2007) Siderophore-based iron acquisition and pathogen control. Microbiol Mol Biol Rev 71:413-451 https://doi.org/10.1128/MMBR.00012-07

Minh BQ, Schmidt HA, Chernomor O, Schrempf D, Woodhams MD, von Haeseler A, Lanfear R (2020) IQTREE 2: New models and efficient methods for phylogenetic inference in the genomic era. Mol Biol Evol 37:1530-1534 https://doi.org/10.1093/molbev/msaa015

Mukherjee S et al. (2017) 1,003 reference genomes of bacterial and archaeal isolates expand coverage of the tree of life. Nat Biotechnol 35:676-683 https://doi.org/10.1038/nbt.3886

Murooka Y (2016) Acetic acid bacteria in production of vinegars and traditional fermented foods. In: Matsushita K, Toyama H, Tonouchi N, Okamoto-Kainuma A (eds) Acetic Acid Bacteria: Ecology and Physiology. Springer Japan, Tokyo, pp 51-72. https://doi.org/10.1007/978-4-431-55933-7_2

Navarro-Muñoz JC, Collemare J (2020) Evolutionary histories of type III polyketide synthases in fungi. Front Microbiol https://doi.org/10.3389/fmicb.2019.03018

Oves-Costales D, Kadi N, Challis GL (2009) The long-overlooked enzymology of a nonribosomal peptide synthetase-independent pathway for virulence-conferring siderophore biosynthesis. Chem Comm 65306541 https://doi.org/10.1039/B913092F

Pandey SS, Patnana PK, Rai R, Chatterjee S (2017) Xanthoferrin, the $\alpha$-hydroxycarboxylate-type siderophore of Xanthomonas campestris pv. campestris, is required for optimum virulence and growth inside cabbage. Mol Plant Pathol 18:949-962 https://doi.org/10.1111/mpp.12451

Pappenberger G, Hohmann H-P (2014) Industrial Production of 1-Ascorbic Acid (Vitamin C) and d-Isoascorbic Acid. In: Zorn H, Czermak P (eds) Biotechnology of Food and Feed Additives. Springer Berlin Heidelberg, Berlin, Heidelberg, pp 143-188. https://doi.org/10.1007/10_2013_243 
Parte AC, Sardà Carbasse J, Meier-Kolthoff JP, Reimer LC, Göker M (2020) List of Prokaryotic names with Standing in Nomenclature (LPSN) moves to the DSMZ. Int J Syst Evol Microbiol https://doi.org/10.1099/ijsem.0.004332

Rahmanpour R, Bugg TDH (2013) Assembly in vitro of Rhodococcus jostii RHA1 encapsulin and peroxidase DypB to form a nanocompartment. FEBS J 280:2097-2104 https://doi.org/10.1111/febs.12234

Reis VM, Teixeira KRdS (2015) Nitrogen fixing bacteria in the family Acetobacteraceae and their role in agriculture. J Basic Microbiol 55:931-949 https://doi.org/10.1002/jobm.201400898

Robinson SL, Christenson JK, Wackett LP (2019) Biosynthesis and chemical diversity of $\beta$-lactone natural products. Nat Prod Rep 36:458-475 https://doi.org/10.1039/C8NP00052B

Roh SW et al. (2008) Phylogenetic characterization of two novel commensal bacteria involved with innate immune homeostasis in Drosophila melanogaster. Appl Environ Microbiol 74:6171-6177 https://doi.org/10.1128/aem.00301-08

Sáenz JP, Grosser D, Bradley AS, Lagny TJ, Lavrynenko O, Broda M, Simons K (2015) Hopanoids as functional analogues of cholesterol in bacterial membranes. PNAS 112:11971-11976 https://doi.org/10.1073/pnas.1515607112

Schaefer AL, Harwood CS, Greenberg EP (2018) "Hot stuff": The many uses of a radiolabel assay in detecting acyl-homoserine lactone quorum-sensing signals. Methods Mol Biol 1673:35-47 https://doi.org/10.1007/978-1-4939-7309-5_3

Schneiker S et al. (2007) Complete genome sequence of the myxobacterium Sorangium cellulosum. Nat Biotechnol 25:1281-1289 https://doi.org/10.1038/nbt1354

Schöner TA et al. (2016) Aryl polyenes, a highly abundant class of bacterial natural products, are functionally related to antioxidative carotenoids. ChemBioChem 17:247-253 https://doi.org/10.1002/cbic.201500474

Schuster M, Sexton DJ, Diggle SP, Greenberg EP (2013) Acyl-homoserine lactone quorum sensing: From evolution to application. Annu Rev Microbiol 67:43-63 https://doi.org/10.1146/annurev-micro-092412155635

Seemann T (2014) Prokka: rapid prokaryotic genome annotation. Bioinformatics 30:2068-2069 https://doi.org/10.1093/bioinformatics/btu153

Seitz M, Klebensberger J, Siebenhaller S, Breuer M, Siedenburg G, Jendrossek D, Hauer B (2012) Substrate specificity of a novel squalene-hopene cyclase from Zymomonas mobilis. J Mol Catal B: Enzymatic 84:72-77 https://doi.org/10.1016/j.molcatb.2012.02.007

Shen B (2003) Polyketide biosynthesis beyond the type I, II and III polyketide synthase paradigms. Curr Opin Chem Biol 7:285-295 https://doi.org/10.1016/S1367-5931(03)00020-6

Siedenburg G, Jendrossek D (2011) Squalene-hopene cyclases. Appl Environ Microbiol 77:3905-3915 https://doi.org/10.1128/aem.00300-11

Sievers M, Swings J (2015) Acetobacteraceae. In: Bergey's Manual of Systematics of Archaea and Bacteria. pp 1-20. https://doi.org/10.1002/9781118960608.fbm00174

Talavera G, Castresana J (2007) Improvement of phylogenies after removing divergent and ambiguously aligned blocks from protein sequence alignments. Syst Biol 56:564-577 https://doi.org/10.1080/10635150701472164

Tanabe T, Funahashi T, Nakao H, Miyoshi S-I, Shinoda S, Yamamoto S (2003) Identification and characterization of genes required for biosynthesis and transport of the siderophore vibrioferrin in Vibrio parahaemolyticus. J Bacteriol 185:6938-6949 https://doi.org/10.1128/jb.185.23.6938-6949.2003

Valera MJ, Mas A, Streit WR, Mateo E (2016) GqqA, a novel protein in Komagataeibacter europaeus involved in bacterial quorum quenching and cellulose formation. Microb Cell Fact 15:88 https://doi.org/10.1186/s12934-016-0482-y

Vu HTL et al. (2019) The traits of the plant growth promoting acetic acid bacterium, Nguyenibacter vanlangensis.Vietnam J Sci Technol 57:439 https://doi.org/10.15625/2525-2518/57/4/13523

Waters CM, Bassler BL (2005) Quorum sensing: Cell-to-cell communication in bacteria. Annu Rev Cell Dev Biol 21:319-346 https://doi.org/10.1146/annurev.cellbio.21.012704.131001

Welander PV, Hunter RC, Zhang L, Sessions AL, Summons RE, Newman DK (2009) Hopanoids play a role in membrane integrity and $\mathrm{pH}$ homeostasis in Rhodopseudomonas palustris TIE-1. J Bacteriol 191:61456156 https://doi.org/10.1128/JB.00460-09

Yamada Y (2016) Systematics of Acetic Acid Bacteria. In: Matsushita K, Toyama H, Tonouchi N, OkamotoKainuma A (eds) Acetic Acid Bacteria: Ecology and Physiology. Springer Japan, Tokyo, pp 1-50. https://doi.org/10.1007/978-4-431-55933-7_1

Yamada Y, Yukphan P, Lan Vu HT, Muramatsu Y, Ochaikul D, Tanasupawat S, Nakagawa Y (2012) Description of Komagataeibacter gen. nov., with proposals of new combinations (Acetobacteraceae). J Gen Appl Microbiol 58:397-404 https://doi.org/10.2323/jgam.58.397 